
problems

M. Taroni

C. J. W. Breward

P. D. Howell

J. M. Oliver 



\title{
Boundary conditions for free surface inlet and outlet problems
}

\author{
M. TARONI, C. J. W. BREWAR D, P. D. H O W E L L, \\ AND J. M. OLIVER \\ Mathematical Institute, University of Oxford, 24-29 St Giles, Oxford OX1 3LB, UK
}

(Received ?; revised ?; accepted ?)

We investigate and compare the boundary conditions that are to be applied to free surface problems involving inlet and outlets of Newtonian fluid, typically found in coating processes. The flux of fluid is a priori known at an inlet, but unknown at an outlet, where it is governed by the local behaviour near the film-forming meniscus. In the limit of vanishing capillary number $C a$ it is well-known that the flux scales with $C a^{2 / 3}$, but this classical result is nonuniform as the contact angle approaches $\pi$. By examining this limit we find a solution that is uniformly valid for all contact angles. Furthermore, by considering the far-field behaviour of the free surface we show that there exists a critical capillary number above which the problem at an inlet becomes over-determined. The implications of this result for the modelling of coating flows are discussed.

Key words:

\section{Introduction}

Free surface fluid inlet and outlet flows are found in a large number of physical situations, most notably industrial coating processes, where a thin film of fluid is coated onto an underlying substrate. Examples include roll coating and screen printing, shown in Figure 1a-b, with many more described in the literature and reviewed by Weinstein \& Ruschak (2004). The distinction is that a free surface forms upstream of an outlet, while it vanishes or 're-forms' downstream of an inlet. A canonical problem that illustrates both flows is that of a bubble slowly displacing a viscous fluid (Figure 1c) considered by Bretherton (1961).

Coating flows were traditionally modelled using lubrication theory (Savage 1982; Wilson 1982), but this approach fails near a forming meniscus, where the flow is essentially two-dimensional (Taylor 1963). More recently it has become possible to solve the full twodimensional coating flow problems numerically (Coyle et al. 1990; Gaskell et al. 1995), but lubrication-based models remain popular because of their simplicity and susceptibility to asymptotic analysis (Carou et al. 2009; Taroni et al. 2012). Furthermore, many of these flows involve a triple-contact-line, which can give rise to a number of mathematical and computational issues. In particular, when a contact line moves with respect to the substrate, this necessarily leads to a non-integrable stress singularity (Dussan V. \& Davis 1974), which must be regularised, for example by adding slip (Hocking 1977).

Film-forming or outlet flows are often 'self-metered', in the sense that the fluid flow and geometry combine to determine the thickness of the film, which is a priori unknown. The question of what boundary conditions to apply within the context of lubrication theory to determine the film thickness and location of the forming meniscus has long-interested 


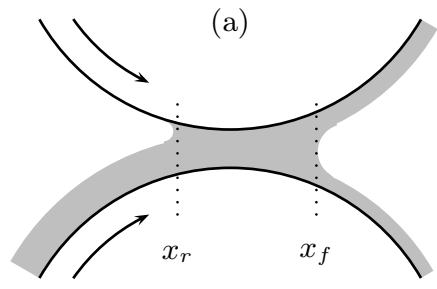

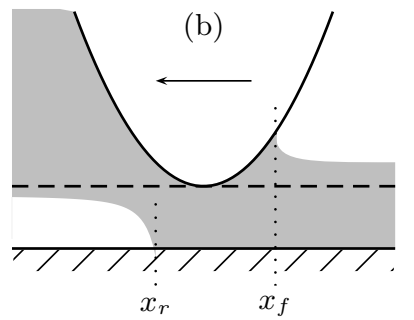

(c)

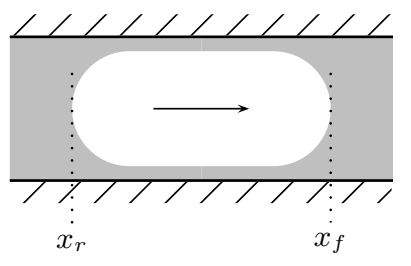

Figure 1: Sketches of (a) forward roll coating and (b) screen printing, examples of coating processes involving inlets and outlets of fluid thin films. Lubrication theory breaks down near forming and re-forming meniscii, which are labelled $x_{f}$ and $x_{r}$ respectively. A canonical problem exhibiting this behaviour is the Bretherton bubble (c).

theoreticians. Most of the early approximate theories were concerned with understanding cavitation in journal bearings and were reviewed by Savage (1977). A formal approach to the problem using matched asymptotic expansions was first presented by Ruschak (1982) in the context of roll coating; this method may be adapted for any film-forming flow but requires solving a local Stokes flow problem, and so simpler approximations remain desirable.

The analogous problem at a fluid inlet has received considerably less attention in the literature because in most practical problems the flow is 'premetered', that is the thickness of the film far upstream is known. At first glance, it would therefore appear that there is one less degree of freedom compared with the outlet problem, leading to an over-determined system when applying the classical Coyne \& Elrod (1970) boundary conditions at a re-forming meniscus (Dowson \& Taylor 1979). In practice, it is often sufficient to 'patch' the two lubrication regions on either side (Moriarty \& Terrill 1996): the local behaviour near the re-forming meniscus is not crucial in determining the global behaviour of the system. However, while this argument may be formally justified in the limit of vanishing capillary number, at higher values lubrication theory-based models for coating processes and numerical solutions to the full problems have been found to disagree (Coyle et al. 1990).

The paper is laid out as follows. We begin in $\S 2$ by analysing a general outlet problem, where a meniscus forms, using matched asymptotic expansions following the example of Ruschak (1982), and extend his analysis to account for a finite contact angle. In $\S 3$ we study the analogous problem at a fluid inlet, and, by considering the far-field behaviour of the free surface, we determine the correct number of boundary conditions that may be imposed. Finally in $\S 4$ we assess the implications of our results to coating flows and propose avenues for future research.

\section{Local problem near a forming meniscus}

\subsection{Governing equations and boundary conditions}

We consider steady two-dimensional flow of a Newtonian fluid of viscosity $\eta$ between two surfaces of length $L$ and arbitrary distance $H(x)=\epsilon L f(x)(\epsilon \ll 1)$ apart, where $f(x)$ varies on an order-one length-scale, with the lower surface (assumed flat for simplicity) moving at speed $U$ in the positive $x$-direction. We assume that a fluid/air interface with surface tension $\gamma$ forms at $x=x_{c}$, and that the capillary number $C a=\eta U / \gamma=\mathcal{O}(1)$. A sketch of the problem is given in Figure 2a. Upstream of the meniscus, the governing Stokes equations may be non-dimensionalised appropriately and, using standard methods of lubrication theory (see, for example, Ockendon \& Ockendon 1995), reduced to the 
(a)
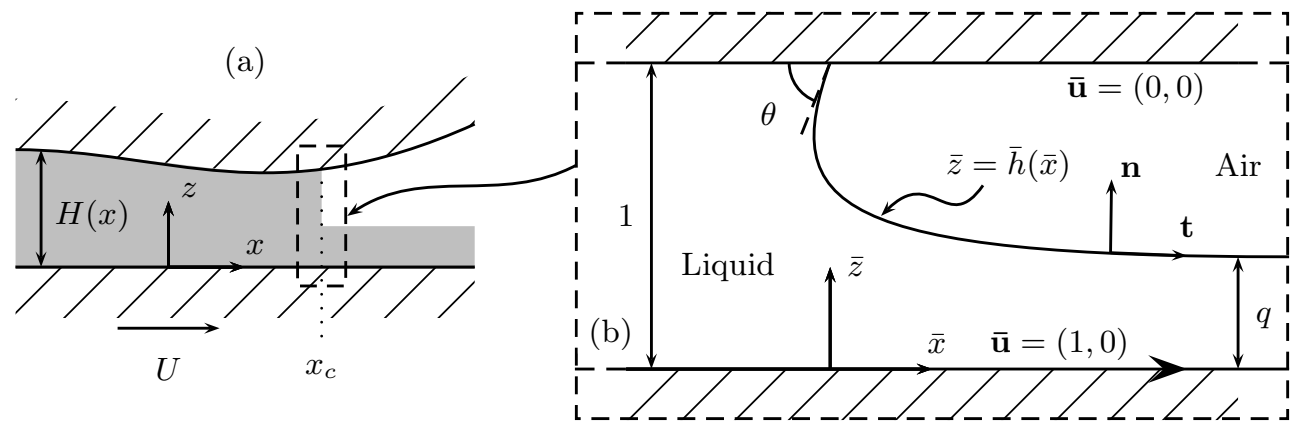

Figure 2: Sketch of a typical outlet problem (a), with a zoom in of the local problem near the forming meniscus shown in (b). The motion of the lower surface is reversed for the case of an inlet with a re-forming meniscus.

leading-order lubrication equations

$$
\begin{aligned}
u & =1-\frac{z}{f}+\frac{p_{x}}{2} z(z-f), \\
w & =\frac{p_{x x}}{4}\left(z^{2} f-\frac{2 z^{3}}{3}\right)+\frac{z^{2} f_{x}}{4}\left(p_{x}-\frac{2}{f^{2}}\right), \\
p_{x} & =\frac{6}{f^{2}}(1-2 \lambda),
\end{aligned}
$$

for the velocity field $(u, w)$ and pressure $p(x), \lambda$ denoting the (constant) horizontal flux.

The pressure is found by integrating (2.1c) subject to an appropriate upstream condition. We then require two additional conditions to find the a priori unknown meniscus position $x_{c}$ and flux $\lambda$. Near $x=x_{c}$ the flow is strictly two-dimensional and the lubrication equations no longer hold. We therefore rescale to an inner region by setting

$$
x=x_{c}+\epsilon \bar{x}, \quad z=f\left(x_{c}\right) \bar{z}, \quad u=\bar{u} \quad w=f\left(x_{c}\right) \bar{w} / \epsilon \quad p=\epsilon \bar{p},
$$

and for clarity denote all inner variables with an overbar. On this length-scale both surfaces are flat to leading order, and the full two-dimensional Stokes flow equations hold in the fluid:

$$
\nabla \bar{p}=\nabla^{2} \overline{\mathbf{u}}, \quad \nabla \cdot \overline{\mathbf{u}}=0 .
$$

The boundary conditions are

$$
\begin{array}{rlrl}
\bar{u}=1, \quad \bar{w} & =0 & \\
\bar{u}=\bar{w}=0 & & \text { on } \bar{z}=0, \\
\overline{\mathbf{u}} \cdot \mathbf{n}=0, & \mathbf{t} \cdot \overline{\boldsymbol{\sigma}} \cdot \mathbf{n}=0, \quad \mathbf{n} \cdot \overline{\boldsymbol{\sigma}} \cdot \mathbf{n}=\frac{1}{C a} \frac{\bar{h}_{\bar{x} \bar{x}}}{\left(1+\bar{h}_{\bar{x}}^{2}\right)^{3 / 2}} & & \text { on } \bar{z}=\bar{h}(\bar{x}), \\
\bar{h}=1, \quad \bar{h}_{\bar{x}}=\tan \theta & \text { at } \bar{x}=0, \\
\bar{h}_{\bar{x}} \rightarrow 0 & \text { as } \bar{x} \rightarrow \infty,
\end{array}
$$

where $\theta$ is the (prescribed) contact angle and $\mathbf{n}, \mathbf{t}$ are the outward unit normal and unit tangent vectors to the free surface $\bar{z}=\bar{h}(\bar{x})$ respectively. A sketch of the local problem is given in Figure 2b. (We note that for film-forming flows not involving a contact line, as found in roll coating or a Bretherton bubble, $\theta=\pi / 2$ and $(2.4 \mathrm{~b})$ is modified to 


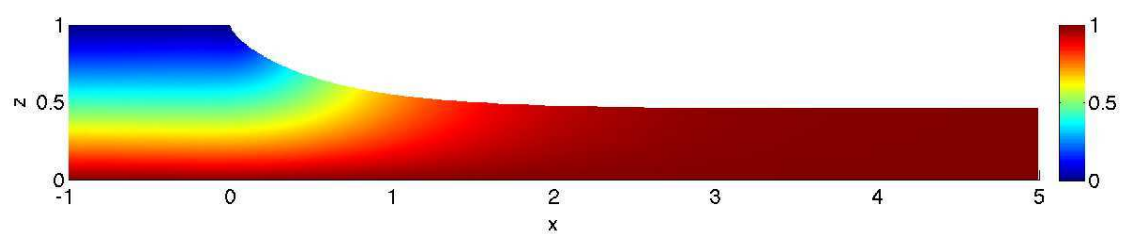

Figure 3: Plot of fluid velocity $|\overline{\mathbf{u}}|$ near forming meniscus, taking $C a=1, \theta=\pi / 2$.

$\bar{u}_{\bar{z}}=\bar{w}=0$ on $\bar{z}=1$.) Finally, matching between the outer lubrication region (2.1) and the inner Stokes flow region (2.3)-(2.4) leads to the boundary conditions

$$
p=0, \quad p_{x}=\frac{6}{f^{2}}(1-2 q) \quad \text { at } x=x_{c},
$$

for the lubrication regime, where the rescaled flux $q(C a, \theta)=\lambda / f\left(x_{c L}\right)$ is uniquely determined as part of the numerical solution to the inner problem, which we discuss in $\S 2.2$.

\subsection{Numerical solution to inner problem}

Equations (2.3)-(2.4) are solved using a finite element ALE formulation implemented using COMSOL. The procedure for generating the finite element counterparts for the weak form of (2.3) using an ALE formulation is well described elsewhere (see, for example, Christodoulou et al. 1997), and so we only note some key points here.

The computational domain is formed by truncating the flow region far upstream and downstream of the meniscus formation point. To apply the force balances (2.3e) on the free surface $\partial \Omega_{f}: y=h(\xi)$ we follow Ruschak (1980) by integrating by parts over the free boundary, thus eliminating the explicit appearance of the surface curvature in the equations:

$$
\int_{\partial \Omega_{f}} \phi \boldsymbol{\sigma} \cdot \mathbf{n} \mathrm{d} s=\frac{1}{C a} \int_{\partial \Omega_{f}} \mathbf{t} \frac{\mathrm{d} \phi}{\mathrm{d} s} \mathrm{~d} s-\frac{1}{C a}[\phi \mathbf{t}]_{a}^{b},
$$

for a piecewise continuous test function $\phi$, where $\partial \Omega_{f} \equiv s \in[a, b]$. The end-point terms in (2.6) can be interpreted physically as shell forces due to surface tension. Appropriate velocity boundary conditions at the inflow of our domain are given by $(2.1 \mathrm{a}, \mathrm{b})$. At the outflow, zero-force boundary conditions, namely $\boldsymbol{\sigma} \cdot \mathbf{n}=0$, were implemented, which are consistent with plug flow. Here we also specified the slope of the free surface, while it was left free at $\bar{z}=0$ : the correct contact angle was imposed by solving iteratively for the flux $q$.

A typical result for the fluid velocity is shown in Figure 3, where we see the flow transition from Couette flow upstream of the forming meniscus to plug flow far downstream. The calculation was performed for a range of contact angles $\theta \in[0, \pi]$ and capillary numbers $C a \in\left[10^{-4}, 10^{2}\right]$, and the results for the flux are shown in Figure 4. At higher values of $C a$, the free surface develops mesh-sized oscillations. We believe these are due to an insufficient mesh refinement away from the contact point, and possible remedies are discussed in $\S 2.4$. We note that our results for $\theta=\pi / 2$ agree with those found using a similar finite element implementation by Hewson (2009) in the range that they investigated, namely $\mathrm{Ca} \in\left[10^{-3}, 1\right]$.

\subsection{Small capillary number limit}

As $C a \rightarrow 0$, the free surface is capillary-static to leading order and tangent to $\bar{z}=\bar{x} \tan \theta$ at the contact point, with radius of curvature $R=1 /(1+\cos \theta)$. By matching this inner solution to a thin 'transition' region in which the Landau-Levich equation holds (see, for 


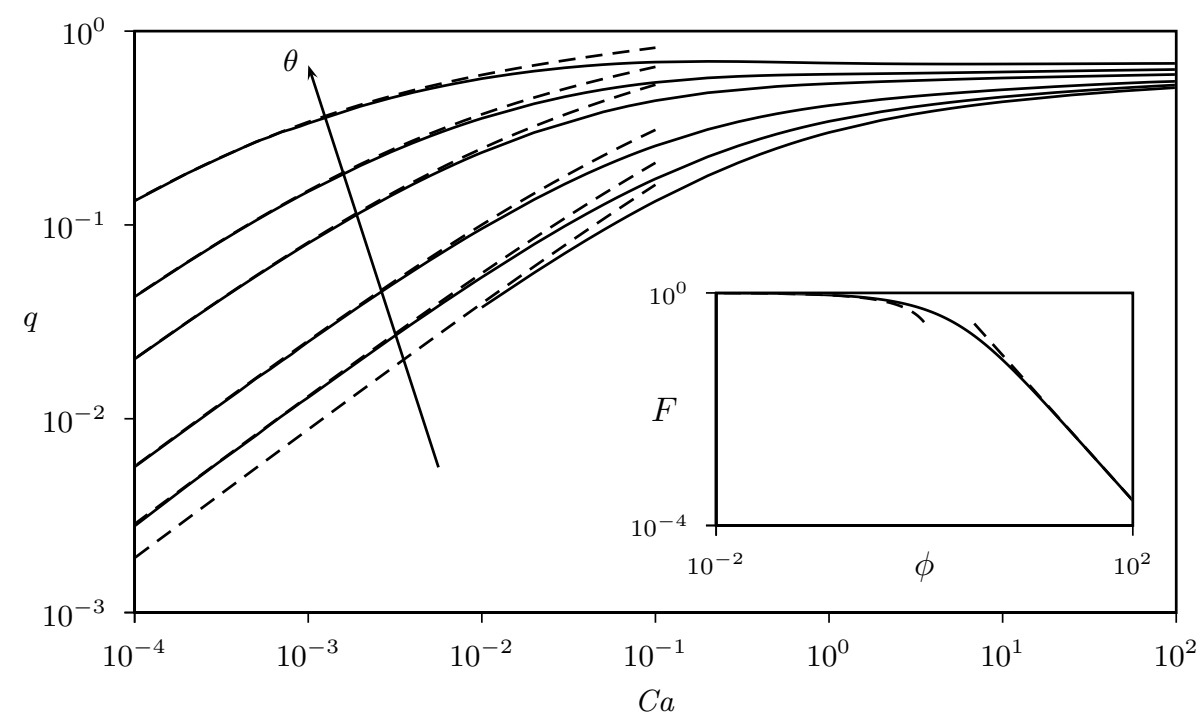

Figure 4: Main plot: numerical results — for the flux $q(\mathrm{Ca})$ for contact angle $\theta / \pi=\{1 / 3,1 / 2,2 / 3,5 / 6,8 / 9,17 / 18\}$. The asymptotic predictions (2.14) are also shown ------ . Inset: plot of $F(\phi)$, with its asymptotic behaviour for $\phi \rightarrow 0$ and $\phi \rightarrow \infty$ plotted ------ .

example, Park \& Homsy 1984), we find

$$
q \sim \frac{A C a^{2 / 3}}{1+\cos \theta},
$$

where $A \approx 1.33757$ is the usual Landau-Levich constant.

We observe that there is a nonuniformity as $\theta \rightarrow \pi$ and the denominator approaches zero. We therefore consider the distinguished limit where $\theta=\pi-C a^{1 / 3} \phi$ with $\phi=\mathcal{O}(1)$, $C a \ll 1$. In this case the fluid domain is uniformly slender and we can use the LandauLevich equation everywhere. Re-scaling by setting

$$
\bar{h}=q H, \quad \bar{x}=q C a^{-1 / 3}\left(X-X_{0}\right),
$$

for arbitrary $X_{0}$ we find that

subject to

$$
\frac{\mathrm{d}^{3} H}{\mathrm{~d} X^{3}}=\frac{3(1-H)}{H^{3}}
$$

$$
\begin{aligned}
& H=\frac{1}{q}, \quad \frac{\mathrm{d} H}{\mathrm{~d} X}=-\phi \quad \text { at } X=X_{0}, \\
& H \rightarrow 1 \quad \text { as } X \rightarrow \infty .
\end{aligned}
$$

The Landau-Levich equation (2.9) has a unique solution satisfying the far-field condition, up to an arbitrary translation which may be fixed by setting

$$
H \sim 1+\mathrm{e}^{-3^{1 / 3} X} \quad \text { as } X \rightarrow \infty .
$$

We can then plot $q=F(\phi)$ parametrically by varying $X_{0}$, as shown in Figure 4 (inset).

We know that $H \sim \frac{1}{2} A X^{2}+B X+C$ as $X \rightarrow-\infty$, where $A$ is the Landau-Levich 
constant. Hence, we get

$$
F(\phi) \sim \frac{2 A}{\phi^{2}} \quad \text { as } \phi \rightarrow \infty
$$

which agrees with (2.7) as $\theta \rightarrow \pi$. At the other extreme we just use the linearisation of (2.11) about $H=1$ to find

$$
F(\phi) \sim 1-\frac{\phi}{3^{1 / 3}} \quad \text { as } \phi \rightarrow 0 .
$$

By combining (2.7) and (2.13) we obtain a uniformly valid solution to $q$ as $C a \rightarrow 0$, namely

$$
q \sim A C a^{2 / 3}\left(\frac{1}{1+\cos \theta}-\frac{2}{(\pi-\theta)^{2}}\right)+F\left(\frac{\pi-\theta}{C a^{1 / 3}}\right) .
$$

This is compared with the numerical results in Figure 4, showing very good agreement for $C a \lesssim 10^{-2}$.

We end this section by noting that for $\pi-\theta \ll 1$, the pressure at the meniscus, given in inner variables by $\bar{p}=1 / R$, is comparable to the upstream lubrication pressure once the reduced capillary number $C a / \epsilon^{3}=\mathcal{O}(1)$. In this case (2.5) no longer holds, and we may instead patch lubrication regions on either side of $x=x_{c}$ together by applying continuity of pressure and flux, as in Moriarty \& Terrill (1996).

\subsection{Large capillary number limit}

For large $C a$, our numerical results suggest that $q(C a) \rightarrow q_{0}=\mathcal{O}(1)$ as $C a \rightarrow \infty$ with $q_{0}$ being independent of $\theta$. This agrees with our expectation that the influence of surface tension is felt only in a vanishingly small region close to the meniscus, and similar results have been found for related problems (Giavedoni \& Saita 1997; Jin et al. 2005). However, better numerical results reaching higher values of $C a$ are required to understand this limit. In particular, it may be preferable to use a boundary element method, which would allow a finer discretisation of the free surface.

\section{Local problem near a re-forming meniscus}

\subsection{Small capillary number limit}

We now reverse the direction of the flow by setting $x \mapsto-x$ (or, equivalently, reverse the direction of the moving boundary) and thus consider an inlet problem in which a fluid/air interface vanishes at $x=x_{c}$. Although in many coating applications the meniscus reattaches to a moving substrate at $x=x_{c}$, leading to a dynamic contact line, here this is not the main point of interest. Indeed we may circumvent it entirely by considering a re-forming meniscus without a contact line, as is the case at the rear of a Bretherton bubble, for example.

The important distinction between an inlet problem and its analogous outlet problem is that the flux $\lambda$ (which is the quantity of interest in many applications) is assumed to be determined by the flow far upstream. In the low- $C a$ limit, this is easily explained by linearising the Landau-Levich equation (2.9) about $H=1$, which leads to

$$
H \sim 1+C_{1} \mathrm{e}^{-3^{1 / 3} X}+C_{2} \mathrm{e}^{3^{1 / 3} X / 2} \cos \left(3^{1 / 3} \sqrt{3} X / 2+C_{3}\right),
$$

for constants $C_{i}$. It is therefore clear that the far-field condition $H \rightarrow 1$ eliminates two free parameters at $+\infty$, but only one at $-\infty$, and so, when performing an asymptotic analysis akin to $\S 2.3$, we should be able to impose the flux in the inlet case, but not in 
the outlet case considered in $\S 2$. It is, however, not obvious how this result generalizes for Stokes flow problems with arbitrary capillary number, and we investigate this further in $\S 3.2$.

\subsection{Far-field analysis}

We consider the canonical problem of steady Stokes flow of a Newtonian fluid between a flat substrate $y=0$ moving at unit speed and a free surface $y=h(x)$. We introduce a streamfunction $\psi$ defined by

$$
u=\frac{\partial \psi}{\partial z}, \quad w=-\frac{\partial \psi}{\partial x}
$$

which satisfies the problem

$$
\begin{array}{rlrl}
\nabla^{4} \psi & =0 & & \text { for } 0<z<h(x), \\
\psi=0, \quad \psi_{z} & =1 & & \text { on } z=0, \\
\mathbf{u} \cdot \mathbf{n}=0, \quad \mathbf{t} \cdot \boldsymbol{\sigma} \cdot \mathbf{n}=0, \quad \mathbf{n} \cdot \boldsymbol{\sigma} \cdot \mathbf{n}=\frac{1}{C a} \frac{h_{x x}}{\left(1+h_{x}^{2}\right)^{3 / 2}} & & \text { on } z=h(x) .
\end{array}
$$

We now linearise about plug flow by seeking solutions of the form

$$
\psi=z+\varepsilon f(z) \exp (m x), \quad h=1+\varepsilon \exp (m x),
$$

where $\varepsilon \ll 1$ and $m \in \mathbb{C}$. The biharmonic equation and boundary conditions (3.3b) are satisfied for

$$
f(z)=A z \sin (m z)+B(\sin (m z)-m z \cos (m z))
$$

for constants $A, B$, and then upon application of the conditions (3.3c) at $z=h(x)$ we find that there are no nontrivial solutions unless

$$
g(m) \equiv \frac{1}{C a}(\cos m \sin m-m)+2\left(m^{2}-\cos ^{2} m\right)=0 .
$$

In Figure 5 we plot the contours of $\Re(g), \Im(g)=0$ for different values of $C a$, showing how the roots of (3.6) evolve. The far-field behaviour of the solution to the free-surface problem (3.3) is determined by the solutions of (3.6) with smallest real part. Although (3.6) was reported by Reinelt \& Saffman (1985), they only considered the behaviour of the negative real root; here we concentrate on the roots with positive real part.

Taking the limit $C a \rightarrow 0$ we find that the smallest roots approximately satisfy

$$
m^{3} \sim-3 C a
$$

which reproduces the thin film scenario (3.1), in which there are two roots with positive real part and one with negative real part. However, as $C a \rightarrow \infty$ we find that

$$
g(m) \sim 2\left(m^{2}-\cos ^{2} m\right),
$$

and so the roots are symmetric about $\Re(m)=0$. This is to be expected since Stokes flow without surface tension is completely reversible.

We find that the transition from there being two smallest roots with $\Re(m)>0$ to just one occurs at the critical value

$$
C a_{c}=\frac{1}{\pi}
$$

The local behaviour of the relation (3.6) near this critical value is approximated by the cubic

$$
\left(m-\frac{\pi}{2}\right)^{3} \sim-\frac{3}{4} \pi^{2}\left(C a-C a_{c}\right)
$$




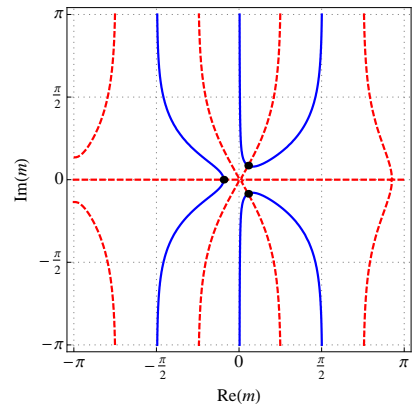

(a) $C a=0.01$

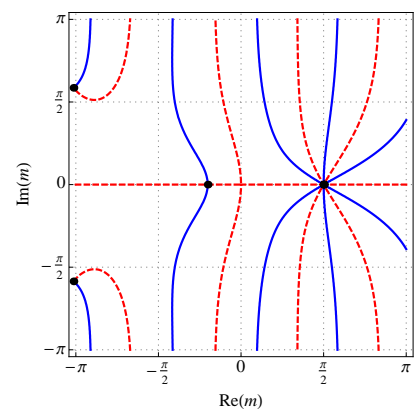

(d) $C a=1 / \pi$

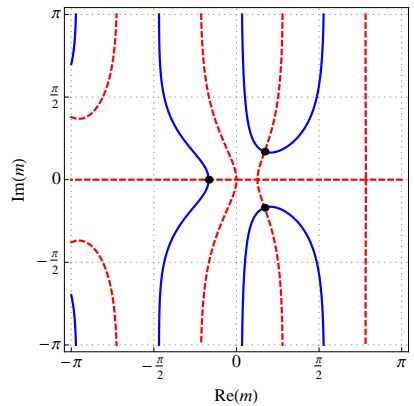

(b) $C a=0.1$

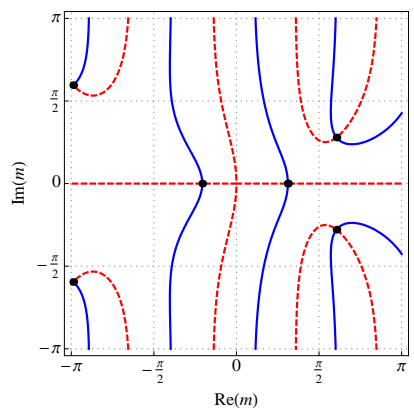

(e) $C a=0.4$

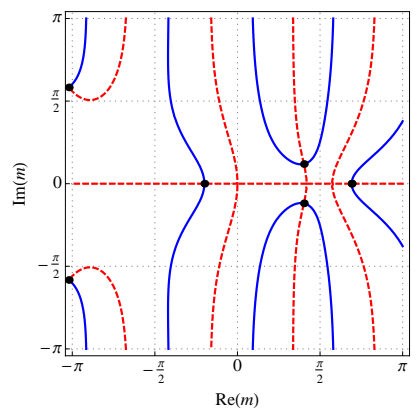

(c) $C a=0.3$

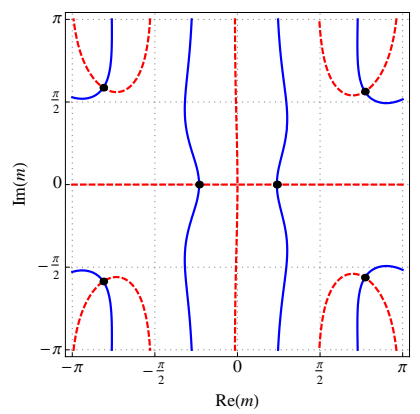

(f) $C a=5$

Figure 5: Contours of $\Re(g)=0 \longrightarrow$ and $\Im(g)=0------$ of the function $g(m)$ for $C a=\{0.01,0.1,0.3,1 / \pi, 0.4,5\}$. The roots of $g(m)$ are marked ' $\bullet$ '.

from which we see that a real root crosses $\pi / 2$ as $C a$ crosses $C a_{c}$. This is illustrated in Figure 5, where we see how at $C a=C a_{c}$ the two complex roots with smallest positive real part coalesce with a real root, which then takes over as the smallest root for $\mathrm{Ca}>\mathrm{Ca}$.

\subsection{Discussion}

The result presented in the previous section explains the disappearance of capillary waves at the rear meniscus of a Bretherton bubble, found numerically for "values of $\mathrm{Ca}$ larger than 0.3" (Giavedoni \& Saita 1999). Furthermore, it shows that we may no longer consider only the smallest eigenvalues to get the correct asymptotic behaviour as $x \rightarrow \infty$. Once the roots coalesce we lose a degree of freedom: if only the smallest eigenvalue is considered, for $\mathrm{Ca}>C a_{c}$ the far-field solution in a reforming meniscus is uniquely determined up to translation (but different from the corresponding solution at a forming meniscus for finite $\mathrm{Ca}$ ).

For the Bretherton problem, for example, this suggests that we can no longer treat the front and rear meniscii separately, feeding in the leading-order flux from the front meniscus to the rear one. The shape of the rear meniscus will depend on exponentially small corrections from the front meniscus, and this suggests a weak dependence of the flux/pressure relationship upon the length of the bubble. Numerical results in the literature are inconclusive in this respect: Fujioka \& Grotberg (2004) reported a weak dependency on the bubble length at $C a=0.4$, but Campana et al. (2007) claimed the opposite. Given that both sets of authors investigated relatively short bubbles, we believe that a more detailed asymptotic and numerical study is required to understand this phenomenon more fully. 
For more general coating problems, we argue that a naive lubrication-type approach, patching across re-forming meniscii and requiring only the local behaviour near forming meniscii to determine the global flux (and hence thickness of deposited film), is no longer justified once $\mathrm{Ca}>C a_{c}$, since the local problem near a re-forming meniscus becomes over-determined. Instead, the flux should be found through a global analysis of the entire coating process.

This result is consistent with our intuition that surface tension influences a vanishingly small region as $C a \rightarrow \infty$. Indeed, when solving coating problems numerically the contact angle can eventually be allowed to select itself (Christodoulou et al. 1997). Although it is perhaps surprising that the transition occurs at such a low value of $C a$, we note that even in the limit of vanishing Capillary number the inlet problem is significantly more delicate than the outlet one and can give rise to a complicated asymptotic structure (Wilson \& Jones 1983). Furthermore, numerical evidence for a change in behaviour at moderate values of $C a$ was given for the case of roll coating by Coyle et al. (1990), who found that $x_{r}$ and $x_{f}$ move closer together as $C a$ increases, so that lubrication theory is no longer valid for the fluid between them.

\section{Conclusions}

The formation of a thin liquid film is a problem of great interest as the flow in the vicinity of the meniscus determines the fluid flux far downstream, which is crucial in many coating applications. The correct procedure to analyse this problem was first performed by Ruschak (1982) in the context of roll coating, but it is time-consuming and needs to be adapted according to the exact geometry. Approximate boundary conditions such as those by Reynolds (1886), Coyne \& Elrod (1970) and, more recently, Hewson et al. (2009) are thus likely to remain popular as they often give sufficiently accurate results. While we have extended the classical result that the flux $q \sim C a^{2 / 3}$ as $C a \rightarrow 0$ to account for the contact angle, a full understanding of the limit $C a \rightarrow \infty$ remains open and warrants further attention.

Inlet problems involving the re-formation of a fluid/air interface are generally less interesting as in most practical situations the local flow does not affect the global behaviour of the system. However, we find that there is a critical value of the capillary number above which the local problem appears to become over-determined, indicating that it may no longer be treated in isolation. In such situations, we believe that a naive decomposition of the problem using lubrication theory is no longer justified, and the full problem must be solved numerically, with the flux found globally as part of the solution.

This publication was based on work supported in part by Award No KUK-C1-013-04, made by King Abdullah University of Science and Technology (KAUST). In addition, the authors are grateful to EPSRC and Du Pont (UK) Ltd. for their financial support via grant CASE 2006/015.

\section{REFERENCES}

Bretherton, F. P. 1961 The motion of long bubbles in tubes. J. Fluid Mech. 10, 166-188.

Campana, D. M., Ubal, S., Giavedoni, M. D. \& Saita, F. A. 2007 Stability of the steady motion of a liquid plug in a capillary tube. Ind. Eng. Chem. Res. 46, 1803-1809.

Carou, J. Q., Wilson, S. K., Mottram, N. J. \& Duffy, B. R. 2009 Asymptotic and numerical analysis of a simple model for blade coating. J. Engrg. Math. 63, 155-176.

Christodoulou, K. N., Kistler, S. F. \& Schunk, P. R. 1997 Advances in computational methods for free surface flows. In Liquid film coating. Scientific principles and their tech- 
nological implications (ed. S. F. Kistler \& P. M. Schweizer), pp. 297-366. Chapman \& Hall.

Coyle, D. J., Macosko, C. W. \& Scriven, L. E. 1990 The fluid dynamics of reverse roll coating. AIChE J. 36 (2), 161-174.

Coyne, J. C. \& Elrod, H. G. 1970 Conditions for the rupture of a lubrication film. Part 1. Theoretical model. ASME J. Lubr. Technol. 92, 451.

Dowson, D. \& TaYlor, C. M. 1979 Cavitation in bearings. Ann. Rev. Fluid Mech. 11, 35-66.

Dussan V., E. B. \& Davis, S. H. 1974 On the motion of a fluid-fluid interface along a solid surface. J. Fluid Mech. 65, 71-95.

Fujioka, H. \& Grotberg, J. B. 2004 Steady propagation of a liquid plug in a two-dimensional channel. J. Biomech Eng-T ASME 126, 567-577.

Gaskell, P. H., Savage, M. D., Summers, J. L. \& Thompson, H. M. 1995 Modelling and analysis of meniscus roll coating. J. Fluid Mech. 298, 113-137.

Giavedoni, M. D. \& Saita, F. A. 1997 The axisymmetric and plane cases of a gas phase steadily displacing a Newtonian liquid - A simultaneous solution of the governing equations. Phys. Fluids 19, 2420-2428.

Giavedoni, M. D. \& Saita, F. A. 1999 The rear meniscus of a long bubble steadily displacing a Newtonian liquid in a capillary tube. Phys. Fluids 11, 786-794.

Hewson, R. W. 2009 Free surface model derived from the analytical solution of Stokes flow in a wedge. J. Fluid Eng. 131, 041205.

Hewson, R. W., Kapur, N. \& Gaskell, P. H. 2009 A model for film-forming with Newtonian and shear-thinning fluids. J. Non-Newtonian Fluid Mech. 162, 21-28.

Hocking, L. M. 1977 A moving fluid interface. Part 2. The removal of the force singularity by a slip flow. J. Fluid Mech. 79 (2), 209-229.

Jin, B., Acrivos, A. \& Münch, A. 2005 The drag-out problem in film coating. Phys. Fluids 19, 103603.

Moriarty, J. A. \& Terrill, E. L. 1996 Mathematical modelling of the motion of hard contact lenses. Euro. J. Appl. Math. 7, 575-594.

Ockendon, J. R. \& OCKendon, H. 1995 Viscous flow. Cambridge University Press.

Park, C.-W. \& Homsy, G. M. 1984 Two-phase displacement in Hele-Shaw cells: theory. J. Fluid Mech. 139, 291-308.

Reinelt, D. A. \& Saffman, P. G. 1985 The penetration of a finger into a viscous fluid in a channel and tube. SIAM J. Sci. Stat. Comput. 6 (3), 542-561.

REYNOLDS, O. 1886 On the theory of lubrication and its application to Beauchamp Tower's experiments, including an experimental determination of the viscosity of olive oil. Philos. Trans. R. Soc. London Ser. A 177, 157-233.

Ruschak, K. J. 1980 A method for incorporating free boundaries with surface tension in finite element fluid flow simulators. Internat. J. Numer. Methods Engrg, 15 (5), 639-648.

RuschaK, K. J. 1982 Boundary conditions at a liquid/air interface in lubrication flows. J. Fluid Mech. 119, 107-120.

SAvage, M. D. 1977 Cavitation in lubrication. Part 1. On boundary conditions and cavity-fluid interfaces. J. Fluid Mech. 80, 743-755.

Savage, M. D. 1982 Mathematical models for coating processes. J. Fluid Mech. 117, 443-455.

Taroni, M., Breward, C. J. W., Howell, P. D., Oliver, J. M. \& Young, R. J. S. 2012 The screen printing of a power-law fluid. J. Engrg. Math. 73, 93-119.

TAYlor, G. I. 1963 Cavitation of a viscous fluid in narrow passages. J. Fluid Mech. 16 (4), 595-619.

Weinstein, S. J. \& Ruschak, K. J. 2004 Coating flows. Annu. Rev. Fluid Mech. 36, 29-53.

Wilson, S. D. R. 1982 The drag-out problem in film coating theory. J. Engrg. Math. 16, 209-221.

Wilson, S. D. R. \& Jones, A. F. 1983 The entry of a falling film into a pool and the airentrainment problem. J. Fluid Mech. 128, 219-230. 



\section{RECENT REPORTS}

12/15 Spreading Speed, Traveling Waves, and Minimal Domain Size in Lewis Impulsive Reaction-diffusion Models

$\mathrm{Li}$

12/16 MCMC methods for functions modifying old algorithms to make Cotter them faster

Roberts

Stuart

White

12/17 Weyl Geometry and the Nonlinear Mechanics of Distributed Point Yavari Defects

Goriely

12/18 A note on oblique water entry

Moore

Howison

Ockendon

Oliver

12/19 Calculus on surfaces with general closest point functions

März

Macdonald

12/20 Multiple equilibria in a simple elastocapillary system

Taroni

Vella

12/21 Multiphase modelling of vascular tumour growth in two spatial dimensions

Hubbard

Byrne

12/22 Chebfun and Numerical Quadrature

Hale

Trefethen

12/23 Moment-based formulation of NavierMaxwell slip boundary conditions for lattice Boltzmann simulations of rarefied flows in microchannels

12/24 Correspondence between one- and two-equation models for solute transport in two-region heterogeneous porous media

Reis

Dellar

Davit

Wood

Debenest

Quintard

12/25 Rolie-Poly fluid flowing through constrictions: Two distinct instabilities

Reis

Wilson

12/26 Age related changes in speed and mechanism of adult skeletal muscle stem cell migration

Collins-Hooper

Woolley

Dyson

Patel

Potter

Baker

Gaffney

Maini

Dash

Patel

12/27 The interplay between tissue growth and scaffold degradation in

ODea engineered tissue constructs

Osborne

El Haj

Byrne

Waters 
12/30 A theoretical investigation of the effect of proliferation and adhe-

Mirams sion on monoclonal conversion in the colonic crypt

Fletcher

Maini

Byrne

12/31 Convergent evolution of spiny mollusk shells points to elastic energy minimum

Chirat

Moulton

Shipman

Goriely

12/32 Three-dimensional oblique water-entry problems at small deadrise angles

Moore

Howison

Ockendon

Oliver

12/33 Second weak order explicit stabilized methods for stiff stochastic

Abdulle differential equations

Vilmart

Zygalakis

12/34 The sensitivity of Graphene ‘Snap-through' to substrate geometry Wagner

Vella

12/35 The physics of frost heave and ice-lens growth

Peppin

Style

12/36 Finite Element Simulation of Dynamic Wetting Flows as an Inter-

Sprittles face Formation Process

Shikhmurzaev

12/37 The Dynamics of Liquid Drops and their Interaction with Solids of

Sprittles Varying Wettabilities

Shikhmurzaev

12/38 Dispersal and noise: Various modes of synchrony in ecological

Bressloff oscillators

Lai

Copies of these, and any other OCCAM reports can be obtained from:

Oxford Centre for Collaborative Applied Mathematics Mathematical Institute

24 - 29 St Giles'

Oxford

OX1 3LB

England

www.maths.ox.ac.uk/occam 